\title{
Downstream Perforations for the Reduction of Turbulence-Aerofoil Interaction Noise: Part II - Theoretical Investigation
}

\author{
Matthew J. Priddin* and Lorna J. Ayton $\dagger^{\dagger}$ \\ University of Cambridge, Cambridge, UK CB3 OWA \\ Sergi Palleja-Cabre ${ }^{\ddagger}$, Paruchuri Chaitanya ${ }^{\S}$ and Phillip Joseph ${ }^{\text {II }}$ \\ University of Southampton, Southampton, UK SO17 1BJ
}

\begin{abstract}
This paper presents a theoretical study of introducing a single gap or slot downstream of the leading edge of a thin rigid plate on aeroacoustic scattering. This is investigated as an elementary model for understanding the effect of downstream perforations on gust-aerofoil interaction noise. Across the slot it is supposed there is zero pressure jump, and a wake is shed from the upstream slot edge such that an unsteady Kutta condition is satisfied. The resulting mixed boundary value problem is reformulated as a Wiener-Hopf problem, and reduced to a singular integral equation suitable for numerical solution. Wave components of the solution may be identified, and plausibly related to diffracted fields by each junction. Theoretical results are compared to experimental results considering sets of perforations. The model is found to be in line with overall qualitative trends in the change in far-field sound power level observed experimentally, but does not account for oscillations from this trend.
\end{abstract}

\section{Nomenclature}

$\phi \quad=$ velocity potential

$p \quad=$ pressure

$c \quad=$ speed of sound

$U_{c} \quad=$ convection speed of vorticity

$M \quad=$ Mach number

$f \quad=$ frequency

$\omega=$ angular frequency $\omega=2 \pi f$

$k=$ acoustic wavenumber $k=\omega / c=2 \pi f / c$

$x, y=$ physical coordinates

$l_{0} \quad=$ distance from leading edge to upstream slot edge

$l_{d} \quad=$ distance from leading edge to downsteam slot edge

$l \quad=$ slot length

$D \quad=$ far-field directivity

$\theta \quad=$ observer angle to flow direction

$\alpha=x$-Fourier transform variable

SPL $=$ sound pressure level

$\mathrm{PWL}=$ sound power level

\footnotetext{
*Graduate Student, Department of Applied Mathematics and Theoretical Physics, Student Member AIAA.mjp98@cam.ac.uk

†EPSRC Early Career Fellow, Department of Applied Mathematics and Theoretical Physics. 1.j . ayton@damtp.cam.ac.uk

${ }^{\ddagger}$ Research fellow, Sergi.Palleja-Cabre@soton.ac.uk

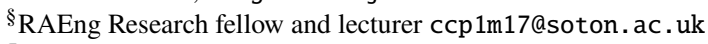

IIProfessor of acoustics, ISVR, pfj@soton.ac.uk
} 


\section{Introduction}

$\mathrm{T}$ HIS paper studies the effect of introducing a slot downstream of a leading edge on the aeroacoustic noise generated due to the scattering of a convected disturbance. This model is investigated with view to understanding more general leading edge modifications consisting of sets of slots or perforations. As an elementary adaptation to the geometry of the leading edge this problem offers an interesting reference point from which to understand opportunities for novel aerofoil adaptions, as well as the aeroacoustic properties of existing devices which may share a similar geometry. It is anticipated that under certain operating conditions the interaction between the sound generated at the leading edge and that from the slot in such a way that the far-field noise radiated is reduced, as has been found in the companion experimental study [Control ID: 3515636]; this paper (Part II of this work) studies an idealized aeroacoustic scattering model accounting for a simple linear interaction between the slot and the acoustic field to understand the effects that may be anticipated within such a framework.

Investigating aerofoil adaptations to reduce noise generated by aerofoils interacting with unsteady flow continues to be a topic of significant interest, with work motivated by the industrial and social imperatives to decrease the environmental impact of noise pollution produced by commercial air travel and wind turbines. To date passive adaptations include the use of material changes such as porosity [1-3] or poroelasticity [4] and geometric changes including serrations [5, 6] and slits [7]. The practical viability of any quiet aerofoil design rests upon its simultaneous aeroacoustic and aerodynamic performance. However, as one might expect, these aeroacoustic benefits typically come at the expense of the core aerodynamic function. The experimental work [Part I - Control ID: 3515636] introduces the use of a small spanwise array of slots located in the vicinity of the leading edge. This may be anticipated to have a smaller aerodynamic penalty due to the small open fraction by comparison with completely porous leading edges, whilst experimental tests indicate that appreciable noise reductions are maintained. This motivates the development of a theoretical understanding of these results. The basic problem considered here is presented in figure 1. A thin plate is supposed to extend at $y=0$ from $0<x<\infty$ that is rigid except for a slot located at the interval $l_{0}<x<l_{d}$. We label the junctions 1, 2, and 3 corresponding to the leading edge, upstream slot edge and downstream slot edge respectively. One might readily imagine variations on this problem where a set of slots is considered, or a slot array is modelled as a homogeneous section where an appropriate boundary condition is applied. Importantly such theoretical models enable us to precisely account for scattering and interference effects.
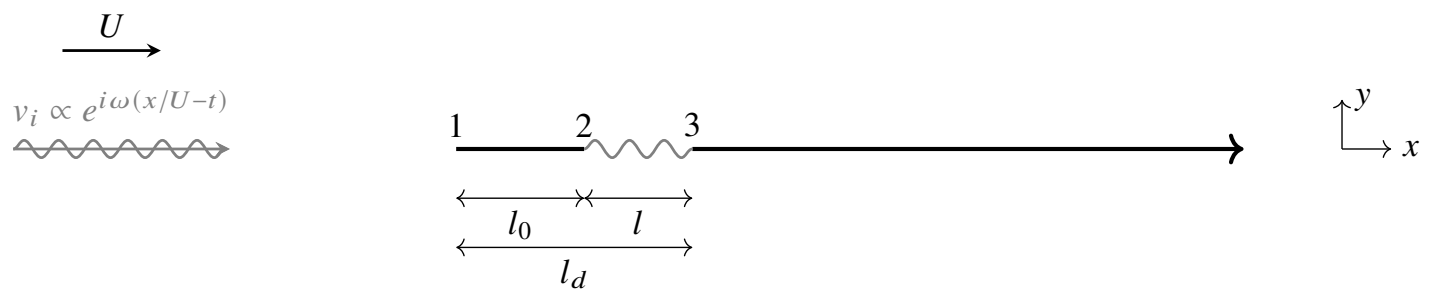

Fig. 1 Schematic side view of gust incident on a slotted leading edge with length scale definitions

To the best of the authors knowledge, the aeroacoustic consequences of locating a slot near a sharp edge have only previously been investigated in relation to trailing edges. In particular, Howe [8] studies the sound generated by turbulent flow over a trailing-edge flap, considering the sound generated by a vortex sheet across the slot and a vortex sheet above the plate. In order to obtain closed form solutions the procedure used in that work is restricted to the regime of a narrow slot $l / l_{0} \ll 1$ and a small modified slot Helmholtz number $k l /\left(1-M^{2}\right) \ll 1$. This corresponds to the most useful limit for the application of interest in the present work. However, practical methods are now available that are not limited in this way; we may thus evaluate the validity of the approximations made and investigate phenomena present in the alternative parameter regimes. The contribution of this work is to apply similar modelling to the distinct physical situation of a leading edge by using a more general solution method that is not restricted to this asymptotic parameter regime.

In our model of a finite length slot, a vortex sheet is allowed to be shed from junction 2 in order for an unsteady Kutta condition to be satisfied there [9, 10]. This is incorporated within an aeroacoustic scattering problem for a otherwise rigid semi-infinite, infinitesimally thin plate in uniform flow. In order to solve the aeroacoustic scattering problem we derive an associated matrix Wiener-Hopf problem [11]. These are amenable to the numerical implementation of the iterative method described in [12, 13]. Here we employ a variation on this method, where we explicitly identify a linear system associated with a discretization based on the numerical implementation in [13], which may be solved directly. 
We so find an expression for the physical fields for which field components may be identified.

In order to maintain analytic tractability, we neglect almost all details of the flow in the vicinity of the slot, which in reality will likely be turbulent and affected by any finite thickness of a real plate. We also do not address the question of reattachment of the supposed vortex sheet at the downstream edge of the slot. These pose a significant challenge to understand analytically, and we anticipate that highly resolved numerical simulations and experimental data would be required to give appropriate insight into these phenomena. Whilst this paper considers the case of a leading edge slot as an elementary adaptation, the approach may have relevance to the situation of high-lift devices and trailing edge slots, for instance extending the work of [8] for trailing edges.

This paper is organized as follows. First, we recall the formulation of scattering a gust by a rigid leading edge and the precise boundary conditions imposed in section III In section IV we present the Wiener-Hopf problems to be solved and briefly describe the numerical approach used. In section $\mathrm{V}$ we discuss the qualitative predictions of the model, consider the question of optimal slot characteristics and compare the theory developed with the complimentary experimental work conducted in [Control ID: 3515636].

\section{Problem}

In this section we formulate the aeroacoustic scattering model, beginning with the governing equation for linearized acoustics about a uniform mean flow, then specifying the boundary conditions to be imposed on the scattered field.

\section{A. Aeroacoustics in uniform mean flow}

We first reduce the problem of acoustics in uniform mean flow to solving the Helmholtz equation to establish the conventions used. Small fluctuations in the pressure, density and velocity potential, indeed any acoustic potential, $\phi$ satisfy the convective wave equation

$$
\left(\left(\frac{\partial}{\partial t}+U \frac{\partial}{\partial x}\right)^{2}-c^{2}\left(\frac{\partial^{2}}{\partial x^{2}}+\frac{\partial^{2}}{\partial y^{2}}+\frac{\partial^{2}}{\partial z^{2}}\right)\right) \phi=0
$$

Here $c$ is the speed of sound. Let $k=\omega / c$ be the acoustic wavenumber associated with the angular frequency $\omega$ and $M=U / c$ the Mach number associated with the uniform background mean flow. By linearity we write the total velocity potential of the disturbance as $\phi_{i}+\phi$, the sum of a prescribed incident field $\phi_{i}$ and a scattered response $\phi$. Whilst we first pose the problem in terms of the velocity potential $\phi$, the physical fields of significance are the normal velocity perturbation

$$
v=\frac{\partial \phi}{\partial y}
$$

and pressure perturbation

$$
p=-\rho\left(\frac{\partial}{\partial t}+U \frac{\partial}{\partial x}\right) \phi .
$$

where $\rho$ is the mean background density. The respective total fields are similarly split into incident and scattered response, $v+v_{i}$ and $p+p_{i}$ respectively.

To consider the leading edge gust-interaction problem we suppose forcing is provided by a velocity perturbation

$$
v_{i}(x, 0, z, t)=e^{i k x / M+i k_{3} z-i \omega t}
$$

at $y=0$ with zero associated pressure jump across $y=0$ (since $k_{1}=k / M$ this disturbance convects with the mean flow it has vanishing associated pressure). The distribution of energy between $k_{3}$ modes for incident convected turbulence may then be characterized by a wavenumber-turbulence spectrum, using a Liepmann or Von Kármán spectrum chosen according to experimental measurements of grid generated turbulence to facilitate comparison with experimental data.

We seek solutions that are harmonic in time $t$ and span $z$ in the form

$$
\phi(\boldsymbol{x}, t)=\mathfrak{R}\left(\tilde{\phi}(x, y) e^{i k_{c} x+i k_{3} z-i \omega t}\right)
$$

where $k_{c}=-k M \beta^{-2}$ with $\beta^{2}=1-M^{2}$. We have included the convective factor $e^{i k_{c} x}$, and then introduce Prandtl-Glauert scaled coordinates as $(\hat{x}, \hat{y})=(x, \beta y), \tilde{\phi}(x, y)=\hat{\phi}(\hat{x}, \hat{y})$ to reduce the governing equation to the Helmholtz equation

$$
\left(\frac{\partial^{2}}{\partial \hat{x}^{2}}+\frac{\partial^{2}}{\partial \hat{y}^{2}}+\hat{k}^{2}\right) \hat{\phi}=0
$$


with reduced wavenumber $\hat{k}$ given by

$$
\hat{k}^{2}=\beta^{-2}\left(\beta^{-2} k^{2}-k_{3}^{2}\right)
$$

In this way the 3D scattering problem in mean flow has been simplified to solving the Helmholtz equation in 2D. Since this derived problem coincides with the physical problem for $k_{3}=0$ and $M=0$, and we refer to $\hat{p}, \hat{\phi}$ as a pressure and velocity potential respectively. The reduced pressure $\hat{p}$ and velocity potential $\hat{\phi}$ are related by

$$
\hat{p}=\rho c\left(i k-i k_{c} M-M \frac{\partial}{\partial \hat{x}}\right) \hat{\phi}
$$

and for reference, the reduced and physical problems are further related by

$$
\frac{\partial}{\partial y}=\beta^{-1} \frac{\partial}{\partial \hat{y}}, \quad \frac{\partial}{\partial x}=\frac{\partial}{\partial \hat{x}}
$$

The physical pressure may be recovered by

$$
p(\boldsymbol{x}, t)=\hat{p}(x, \beta y) e^{i k_{c} x+i k_{3} z-i \omega t}
$$

and an incident gust incurs a reduced forcing

$$
\hat{v}_{i}(\hat{x}, 0)=e^{i\left(k / M-k_{c}\right) \hat{x}} .
$$

We solve the reduced problem, and drop hat decorations. For simplicity we restrict attention to the dominant source with $k_{3}=0$.

\section{B. Boundary Conditions}

We now describe the boundary conditions to be imposed. Recall our model consists of a finite slot from $x=l_{0}$ to $x=l_{d}$ in a semi-infinite rigid plate of infinitesimal thickness along $y=0$, extending along $x \geq 0$. We first require the scattered field $\phi$ to satisfy a radiation condition at infinity to ensure it consists only of outgoing waves. On the rigid plate, the total normal velocity must vanish, so the scattered normal velocity must be equal and opposite to the velocity of the incident disturbance. In so doing we suppose that the incident disturbance downstream of the slot is unaffected by the slot. Specific phase or magnitude differences could be supposed, but in the absence of clear information as to realistic distributions, we make this strong simplification. Upstream of the plate we require continuity of normal velocity and pressure. To close the problem we must specify conditions on the slot, $l_{0}<x<l_{d}, y=0$, in terms of $[p]$ and $v$. Herein lies a non-trivial question of this theoretical investigation; what is the appropriate boundary condition to apply along the slot?

For this investigation, we attempt to consider the simplest idealised model for a slot in an infinitesimal rigid plate. We suppose the slot interface is a free surface where we require continuity of particle displacement. In the case of equal uniform flows above and below the interface (as can be assumed for a flat plate at zero angle of attack to the mean flow) this corresponds to equality of the vertical particle velocity above and below the interface. We additionally require continuity of pressure, as the surface cannot support a pressure jump. We allow a vortex sheet to be shed from the upstream edge of the slot in order to regularise the pressure at this edge and so satisfy an unsteady Kutta condition [9. 10. This is naturally imposed in the course of the Wiener-Hopf procedure as discussed later, and noted in previous works [10].

In summary, the boundary conditions at $y=0$ are

$$
\begin{aligned}
{[p](x, 0) } & =0 \\
v(x, 0) & =-v_{i}(x, 0) \\
{[v](x, 0) } & =0 \\
\lim _{x \rightarrow l_{0}^{+}}|p(x, 0)| & <\infty
\end{aligned}
$$

where $[\circ](x, 0)=\circ\left(x, 0_{+}\right)-\circ\left(x, 0_{-}\right)$denotes the jump in o across $y=0$.

We remark that the problem may alternatively be formulated in terms of the velocity potential. Here the one might follow previous works, e.g. [14], and solve two problems in order to satisfy the unsteady Kutta condition: a first solution 
with $[\phi]$ continuous across the slot, and a second solution with a prescribed jump in $\phi$ associated with zero pressure i.e. corresponding to a solution with a wake of defined strength across the slot. The desired solution satisfying the unsteady Kutta condition may then be found as a linear combination of these solutions cancelling the pressure singularity at $x=l_{0}$. In [15] it is suggested that in certain cases it is appropriate to vary this amplitude in order to match the amount of vorticity shed. This could offer a useful insight into the role of the each part of the scattered field, though introduces another empirical parameter to the model.

\section{Scattering problem solution}

We have now described a model for the scattered field due to an incident gust impinging on rigid leading edge with a slot. In this section we derive a Wiener-Hopf equation and give an overview of a method of solution. Further details of the solution approach may be found in appendix A

\section{A. Formulation of the Wiener-Hopf equations}

The starting point for our solution is a spectral equation that relates the Fourier transforms of the unknown boundary data. After performing a $x$-Fourier transform of the governing Helmholtz equation we see that solutions for $\phi$ satisfying the radiation condition take the form

$$
\phi(x, y)=\int_{-\infty}^{\infty} \Phi_{\gtrless}(\alpha) e^{-i \alpha x-\gamma(\alpha)|y|} \mathrm{d} \alpha \quad y \gtrless 0
$$

where $\gamma(\alpha)=(i(\alpha-\hat{k}))^{1 / 2}(-i(\alpha+\hat{k}))^{1 / 2}$ with the branch cuts chosen in the standard manner [11] as rays parallel to the imaginary $\alpha$ axis, and $\Phi_{\gtrless}$ are to be determined. To satisfy $[v]=0$, we require $y$-anti-symmetric solutions, such that $\Phi=\Phi_{>}=-\Phi_{<}$. Using the expression $[13$, we find that the pressure jump and normal velocity of the scattered field at $y=0$ are related by

$$
K(\alpha) \int_{-\infty}^{\infty}[p](x, 0) e^{i \alpha x} d x+\int_{-\infty}^{\infty} v(x, 0) e^{i \alpha x} d x=0
$$

where

$$
K(\alpha)=\frac{\gamma(\alpha)}{2 \mathcal{P}(\alpha)}
$$

with $\mathcal{P}(\alpha)=i\left(k-M k_{c}+M \alpha\right)$. The pole at $\alpha=-k / M+k_{c}$ corresponds to a pressure-free velocity perturbation that convects with the flow.

We see that equation (14) contains only terms which arise in our boundary conditions, namely $[p]$, and $v$ along $y=0$. We therefore insert the known sections of these boundary conditions to generate an equation consisting of unknown terms on the left hand side, and all known terms on the right hand side. On substituting the boundary conditions given in equation (12) into equation (14), we find

$$
\int_{-\infty}^{0} v e^{i \alpha x} d x+K(\alpha) \int_{0}^{l_{0}}[p] e^{i \alpha x} d x+\int_{l_{0}}^{l_{d}} v e^{i \alpha x} d x+K(\alpha) \int_{l_{d}}^{\infty}[p] e^{i \alpha x} d x=F(\alpha) .
$$

Here all unknown terms on the left hand side are evaluated at $(x, 0)$, and $F$ denotes the known terms:

$$
F(\alpha)=\int_{0}^{l_{0}} v_{i} e^{i \alpha x} d x+\int_{l_{d}}^{\infty} v_{i} e^{i \alpha x} d x
$$

Next, we represent the unknown terms in equation (16) by functions $\Psi_{m \pm}(\alpha)$ with known analyticity and growth in the complex plane. As standard in the application of the Wiener-Hopf technique, the wavenumber $k$ is considered to have a small imaginary part to ensure causality. This means that there is a small positive constant $\varepsilon$ such that in the strip 
$-\varepsilon<\operatorname{Im}(\alpha)<\varepsilon$ all functions are analytic. We define

$$
\begin{aligned}
& \int_{-\infty}^{0} v e^{i \alpha x} d x=\Psi_{1-}(\alpha) \\
& \int_{0}^{l_{0}}[p] e^{i \alpha x} d x=e^{i \alpha l_{0}} \Psi_{2-}(\alpha)=\Psi_{1+}(\alpha) \\
& \int_{l_{0}}^{l_{d}} v e^{i \alpha x} d x=e^{i \alpha l_{d}} \Psi_{3-}(\alpha)=\Psi_{2+}(\alpha) \\
& \int_{l_{d}}^{\infty}[p] e^{i \alpha x} d x=e^{i \alpha l_{d}} \Psi_{3+}(\alpha)
\end{aligned}
$$

By their definition in terms of Fourier transforms, $\Psi_{m+}$ are analytic and have at worst algebraic growth in the upper half $\alpha$ plane $\left(\mathrm{UH} \alpha \mathrm{P}=\mathcal{D}_{+}\right) \operatorname{Im}(\alpha)>-\varepsilon$, whilst $\Psi_{m-}$ are analytic and have at worst algebraic growth in the lower half $\alpha$ plane $\left(\mathrm{LH} \alpha \mathrm{P}=\mathcal{D}_{-}\right) \operatorname{Im}(\alpha)<\varepsilon$. We use \pm subscripts to denote functions analytic with at worst algebraic growth in $\mathcal{D}_{ \pm}$.

We have defined a total of 6 unknowns $\boldsymbol{\Psi}_{ \pm}$in equation (18), three of which are analytic in $\mathrm{UH} \alpha \mathrm{P}$ and three analytic in $\mathrm{LH} \alpha \mathrm{P}$. Writing equation (16) in terms of $\boldsymbol{\Psi}_{ \pm}$we form the $3 \times 3$ matrix Wiener-Hopf equation

$$
\boldsymbol{E}_{-} \operatorname{diag}(1, K, 1) \boldsymbol{\Psi}_{-}+\boldsymbol{E}_{+} \operatorname{diag}(K, 1, K) \boldsymbol{\Psi}_{+}=\boldsymbol{F}
$$

for the unknowns $\Psi_{ \pm}=\left(\Psi_{1 \pm}, \Psi_{2 \pm}, \Psi_{3 \pm}\right)^{T}$. Here the matrices $\boldsymbol{E}_{ \pm}$respectively correspond to upper(lower) triangular matrices with entries that are exponentials decaying in the $\mathrm{UH} \alpha \mathrm{P}(\mathrm{LH} \alpha \mathrm{P})$

$$
\boldsymbol{E}_{+}=\left(\begin{array}{ccc}
1 & e^{i \alpha l_{0}} & e^{i \alpha l_{d}} \\
0 & 1 & e^{i \alpha l} \\
0 & 0 & 1
\end{array}\right), \quad \boldsymbol{E}_{-}=\left(\begin{array}{ccc}
1 & 0 & 0 \\
e^{-i \alpha l_{0}} & 1 & 0 \\
e^{-i \alpha l_{d}} & e^{-i \alpha l} & 1
\end{array}\right)
$$

The equation is forced by the incident gust through the RHS

$$
\boldsymbol{F}=F(\alpha)\left(1, e^{-i \alpha l_{0}}, e^{-i \alpha l_{d}}\right)^{T}
$$

The matrix Wiener-Hopf equation (19) is suitable for the numerical procedure described in [12, 13]. Here we perform essentially the same manipulations, but rather than solving the system iteratively, we identify a discretization implicit in that method by introducing a basis for certain unknown values of $\boldsymbol{\Psi}$, and solve directly. The problem reduces to a singular integral equation that may be solved by drawing on the methods in [16, 17]. We defer details of this procedure to appendix A

We find explicit expressions for $\boldsymbol{\Psi}_{ \pm}$in terms of numerically determined coefficients. The $x$-Fourier transform of the pressure jump may then be recovered as

$$
P(\alpha, 0)=\int_{-\infty}^{\infty}[p](x, 0) e^{i \alpha x} d x=\Psi_{1+}(\alpha)+E_{13+}(\alpha) \Psi_{3+}(\alpha) .
$$

from which the acoustic pressure is found as

$$
p(x, y)=\frac{\operatorname{sgn}(y)}{4 \pi} \int_{-\infty}^{\infty} P(\alpha, 0) e^{-|y| \gamma(\alpha)+i k_{c} x-i \alpha x} d \alpha .
$$

In the far field, $r=\sqrt{x^{2}+y^{2}} \rightarrow \infty$, the pressure may be approximated by applying the method of steepest descent (as detailed in [13]). We thus define the far-field directivity, $D(\theta)$, as

$$
|p(r, \theta)| \sim \frac{D(\theta)}{\sqrt{r}}, \quad r \rightarrow \infty,
$$

where $\theta$ is the polar angle measured from the downstream direction. 


\section{B. Solution decomposition by junction}

In this section we propose a decomposition of the pressure field into terms associated with waves radiating from each junction. We first remark that for each term in the expression for the pressure field from equations (22) and (23), we may identify singularities and growth throughout the complex plane. This allows us to decompose the solution into terms with explicit exponential behaviour. These exponential terms arose from the junctions in the boundary value problem as the $x$ locations where Fourier transforms of boundary data are truncated. We find that

$$
P(\alpha, 0)=P_{1}(\alpha)+P_{2}(\alpha) e^{i \alpha l_{0}}+P_{3}(\alpha) e^{i \alpha l_{d}}
$$

where $P_{m}$ decay algebraically as $|\alpha| \rightarrow \infty$. In the spatial inversion (23) the exponential behaviour determines whether we may deform the inversion contour into the upper or lower half plane. By Jordan's lemma, when integrating a decaying function against an exponential factor $e^{i \alpha\left(x_{0}-x\right)-\gamma(\alpha)|y|}$ we may deform into the $\mathrm{UH} \alpha \mathrm{P}$ for $x<x_{0}$ and $\mathrm{LH} \alpha \mathrm{P}$ for $x>x_{0}$. We pick up contributions from the singularities of $f$ in the respective half plane. With our branch cut conventions, these correspond to waves with $x$-wavenumber

$$
\begin{array}{rl}
k(1-M) \beta^{-2} & x>x_{0} \\
-k(1+M) \beta^{-2} & x<x_{0}
\end{array}
$$

for the pressure field. We so infer that such a term corresponds to a wave with phase velocity directed away from $x=x_{0}$. For us this is precisely the pressure wave diffracted from a junction at $x=x_{0}$.

\section{Results}

This section presents the results provided by the theoretical model, first investigating the model in general and then in comparison with the experimental results of [Control ID: 3515636].

\section{A. Example near field}

We first present illustrative results for the fields near the slotted leading edge. We choose $M=0.1, k l_{0}=0.5$, $l_{d} / l_{0}=1.5$. Figure 2 we plot the total scattered pressure, normal and tangential velocity fields. We note that panels (a)

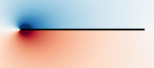

(a) real part of pressure $p$

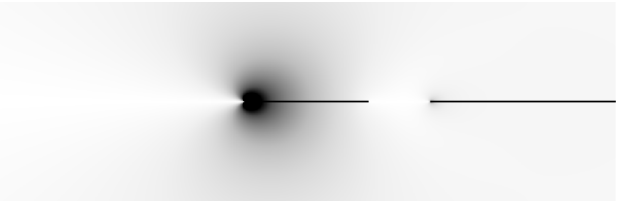

(c) magnitude of pressure $p$

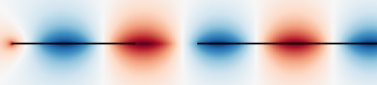

(b) real part of normal velocity $v$

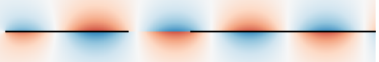

(d) real part of tangential velocity $u$

Fig. 2 Example plots for near-field unsteady scattered pressure and velocity for a slotted leading edge, $k l_{0}=0.5$, $M=0.1$ and $l_{d} / l_{0}=1.5$. Note each panel corresponds to the same arbitrary time instant, but the color scale for each panel is not equal.

and (c) show the anticipated leading edge singularity in the pressure field at junction 1 , as well as a weaker singularity a junction 3. No singularity is observed at junction 2, in line with the imposition of the unsteady Kutta condition. The scattered pressure field vanishes at the slot. The normal velocity is continuous across the slot in panel (b), but the tangential velocity jumps, as seen in panel (d).

\section{B. Effect of slot offset and velocity}

We now investigate the general behaviour of our slotted edge model. The model is found to predict differences in far-field pressure that vary with frequency and flow velocity. From experimental findings in [Control ID: 3515636], 
significant noise reductions are observed that collapse on a hydrodynamic length scale $f l_{d} / U_{c}$, scaling lengths with the distance between junctions 1 and 3. We take $U$ in our model to correspond to the convection velocity of vorticity at the plate so as to more appropriately model the interaction of the disturbance at the scattering junctions. For comparison with experimental setups, this is supposed to be a fraction of the jet velocity, $U_{c}=0.7 U_{\text {jet }}$. Corrections on far-field propagation by supposing this flow speed everywhere are neglected, and anticipated to be small for low Mach number flows of interest here. We restrict attention gusts with $k_{3}=0$ which form the dominant contribution to far-field noise. We present results for the change in Sound Power Level (PWL) between the slot model and a rigid leading edge with no slot, giving an integrated measure of change in far-field pressure over an arc of observer angles in the far-field. Here, where we consider the theoretical model alone, we take this arc to be observer angles $60^{\circ}<\theta<120^{\circ}$ to the downstream flow axis. We plot the change in PWL, $\triangle \mathrm{PWL}$, compared to a semi-infinite rigid (no slot) with sign convention such that positive $\triangle \mathrm{PWL}$ corresponds to a reduction in noise.

Figure 3 presents results for $\triangle \mathrm{PWL}$ scaling frequencies on (a) an acoustic scale and (b) a hydrodynamic scale for $l_{d} / l_{0}=1.1$ at a range of convection velocities $U_{c}$. We observe an appreciable reduction of up to $4 \mathrm{~dB}$ in far-field

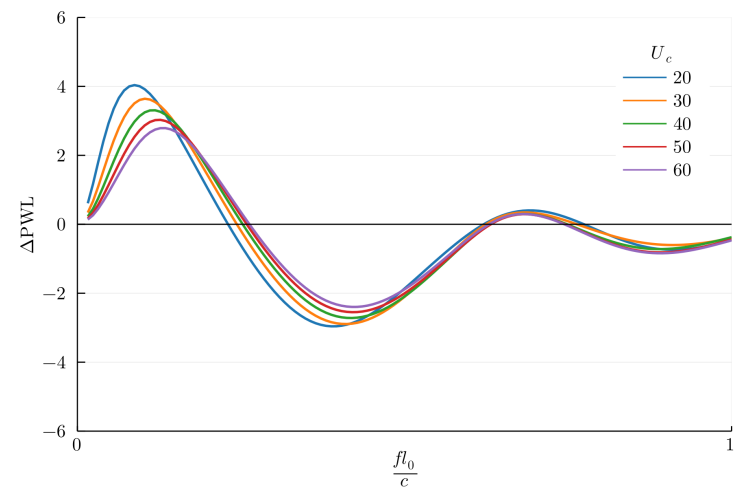

(a) acoustic scaling

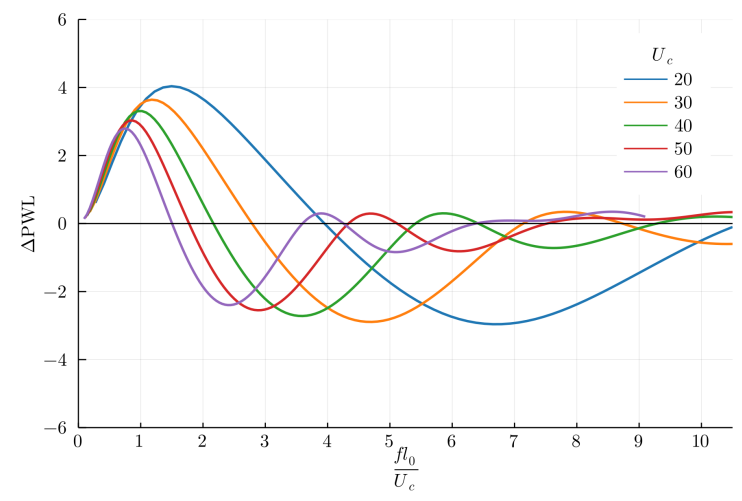

(b) hydrodynamic scaling

Fig. 3 Comparison of $\Delta \mathbf{P W L}$ with velocity scaling, for $l_{d} / l_{0}=1.1$ at $U_{c}=20,30,40,50,60 \mathbf{m s}^{-1}$. Positive $\Delta \mathbf{P W L}$ corresponds to a reduction in far-field noise.

PWL at a low frequencies. The computed $\triangle \mathrm{PWL}$ then fluctuates with increasing frequency with decaying amplitude, leading to an increase in noise of up to $3 \mathrm{~dB}$. These higher frequency fluctuations appear to collapse when scaled on an acoustic length scale with the sound speed and length $l_{0}$ in panel (a). We remark that stronger oscillations in $\triangle \mathrm{SPL}$ are found when considering a particular observer angle, but are suppressed on averaging over an arc. The low frequency behaviour appears to arguably collapse best with $U_{c}$ in panel (b), but neither velocity scale provides a clear collapse for the frequency at which the maximum noise reduction occurs. This indicates that there is not a clear separation between role of the phase difference of the incident hydrodynamic disturbance between junctions, and the back-scattering of the diffracted acoustic wave.

In figure 4 we compare the length scale on which the primary low frequency noise reduction is observed by taking $U_{c}=40 \mathrm{~ms}^{-1}$ and varying $l_{d} / l_{0}$. In panel (a) we scale with $l_{d}$, the distance between junctions 1 and 3 , and in panel (b) we scale with $l_{0}$. the distance between junctions 1 and 2 . We find that scaling on $l_{d}$ provides a better collapse of the low frequency behaviour, but $l_{0}$ provides a better collapse of the higher frequency behaviour. The scaling with $l_{d}$ in line with experimental observations for a set of perforations.

\section{Decomposition of scattered field by junction}

In the hope of gaining some insight into how these changes in far-field noise occur, we consider the decomposition of the scattered field highlighted in section IV.B. We recall that this separates the field into components that separately satisfy the wave equation, up to a discontinuity across $y=0$, and each may be associated with waves with phase velocity strictly away from a particular junction. These so naturally isolate the field diffracted from a particular junction (accounting for multiple scattering).

In figure 5 we compare the magnitude of each component $p_{i}$, for $l_{d} / l_{0}=1.5$ and $M=0.1$ at (a) the frequency of greatest PWL reduction, and (b) the maximum PWL increase. In each case the near field is dominated by the diffracted field from the leading edge, junction 1 . We see that the Kutta condition has suppressed any singularity at junction 2 , 


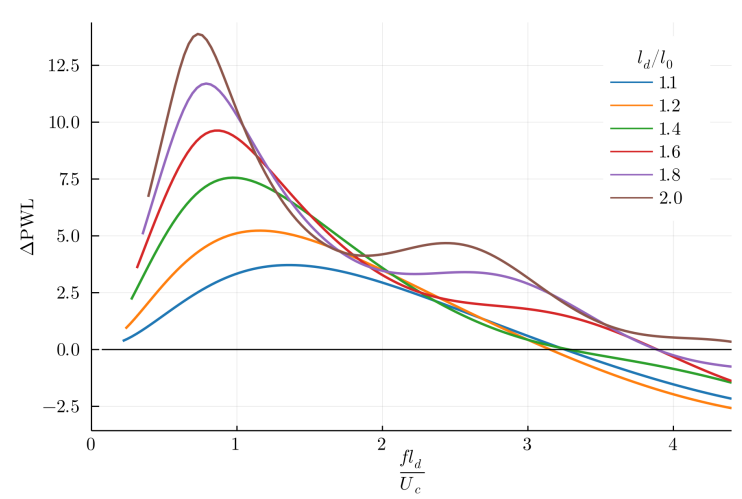

(a) scaling on $l_{d}$

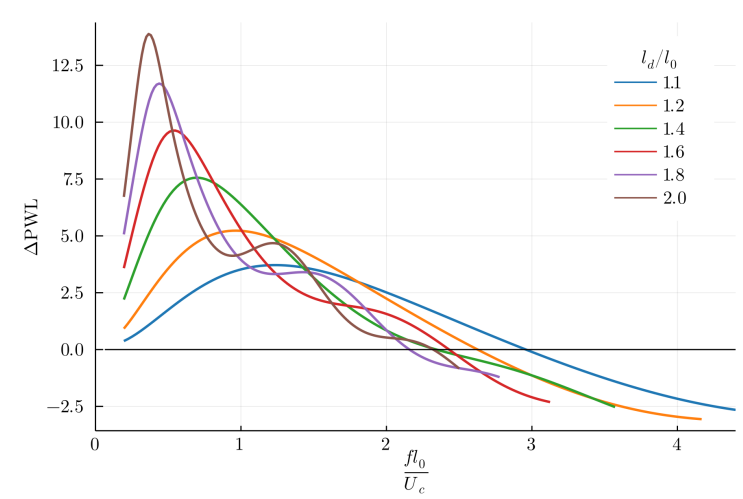

(b) scaling on $l_{0}$

Fig. 4 Comparison of $\Delta \mathbf{P W L}$ with length scaling, for $l_{d} / l_{0}=1.1,1.2,1.4,1.6,1.8,2.0$ at $U_{c}=40 \mathrm{~ms}^{-1}$

and further partially suppressed the singularity at junction 3 . We note that the fields associated with junctions 2 and 3 appear more significant with respect to that of junction 1 in the case of panel (a). This seems consistent with the associated magnitude of $\triangle \mathrm{PWL}$ in this case being appreciably larger.

In figure 6 we compare the phase of the field components for $l_{d} / l_{0}=1.5$ and $M=0.1$ at (a) the frequency of greatest PWL reduction, and (b) the maximum PWL increase. Interestingly, we observe that for maximum noise reduction, the phase of the $p_{1}$ at junction 1 is approximately out of phase with that of $p_{2}$ at junction 2 and $p_{3}$ at junction 3 . This is plausibly consistent with the diffracted fields from junctions 2 and 3 together acting to interfere with the diffracted field from junction 1 to provide the far-field reduction. By contrast, in the case of maximum noise increase, each component $p_{i}$ at the corresponding scattering junction $i$ is approximately in phase, perhaps consistent with each diffracted field constructively interfering to generate a noise increase. Note that moving away from the scattering junction, destructive or constructive interference between fields appears to be dependent on observer location, so these remarks should be taken with caution.

We now turn to the decomposition of the far-field directivity. In figure 7 we plot the far-field directivity as a function of observer angle, for different binary splittings of $D=D_{1}+D_{2}+D_{3}$, for example $\left(D_{1}, D_{2}+D_{3}\right)$. In panels (a) and (c) we find that the $D_{2}$ and $D_{3}$ both act so as to significantly reduce the total far-field directivity, which suggests that neither junction alone may be considered responsible for the noise reductions observed in this model, with interference with scattering from junction 2 appearing to be more significant that from junction 1 . This is consistent with figure 4 where we found that rescaling on neither $l_{d}$ (corresponding to junction 1-3 interaction) or $l_{0}$ (corresponding to junction 1-2 interaction) was sufficient to completely collapse the peak in noise reduction.

\section{Comparison with experimental data}

In this section we compare with the experimental results presented in [Control ID: 3515636], to validate the model. Experiments were conducted in the Institute of Sound and Vibration Research's open-jet wind tunnel facility, using a flat plate with a leading edge insert to facilitate different test cases as described therein. We compare with results between our slot model with experimental results for $D=1 \mathrm{~mm}$ diameter holes arranged within a $T=2.5 \mathrm{~mm}$ spaced square lattice. For ease of comparison with different experimental cases, we take the slot length to be the streamwise length of the slot array, $N T$, where $N$ is the number of hole rows. This means our theoretical model has a larger open area than the corresponding experimental case, and $l_{0}$ and $l_{d}$ do not precisely align with hole edges, which should be borne in mind when interpreting the following results.

In figure 8 we compare $\triangle \mathrm{PWL}$ between the theoretical model and experimental results, where the PWL has been computed by integrating over the array of 16 microphones locations spaced between observer angles $30^{\circ}$ and $130^{\circ}$ approximately $1 \mathrm{~m}$ from the leading edge, as described in [Control ID: 3515636]. We compare cases with $l_{d} / l_{0}=1.1,1.2,1.4$, and $U_{\text {jet }}=40$ and $60 \mathrm{~ms}^{-1}$, supposing these cases of a shorter perforated section being most relevant to the single slot model presented here. In each case $l_{0}$ was equal to $25 \mathrm{~mm}$. We observe some qualitative agreement in the magnitude and overall trend in $\Delta \mathrm{PWL}$, that is a maximum reduction near $f l_{d} / U_{c}=1$, decreasing at higher frequencies. In figure 9 we compare the variation in the change in Sound Pressure Level, $\Delta$ SPL, with frequency for a range of angles. Some qualitative agreement is observed at $40 \mathrm{~ms}^{-1}$ in the trend of $\Delta$ SPL curves as the observer 


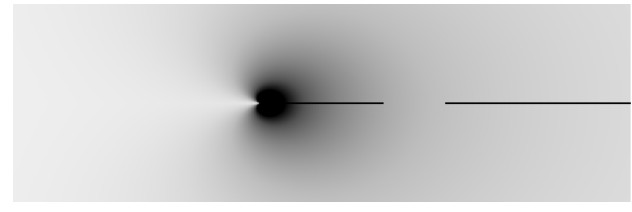

(a) $\max \triangle \mathrm{PWL}$ (noise reduction) - junction 1

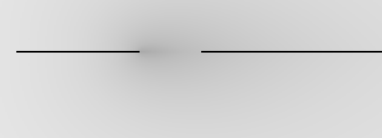

(c) $\max \triangle \mathrm{PWL}$ (noise reduction) - junction 2

(e) $\max \triangle P W L$ (noise reduction) - junction 3

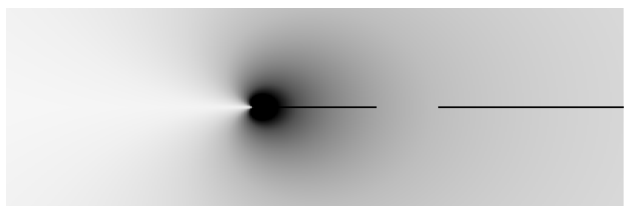

(b) $\min \triangle \mathrm{PWL}$ (noise increase) - junction 1

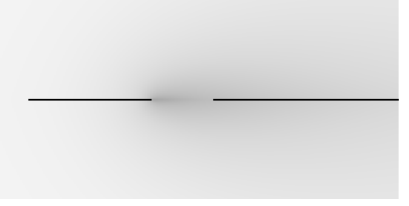

(d) $\min \triangle \mathrm{PWL}$ (noise increase) - junction 2

(f) $\min \triangle \mathrm{PWL}$ (noise increase) - junction 3

Fig. 5 Magnitude of near field pressure field at frequency corresponding to (a) maximum and (b) minimum $\Delta \mathbf{P W L}$ for $l_{d} / l_{0}=1.5, M=0.1$ (colorbar scales equal within each column)

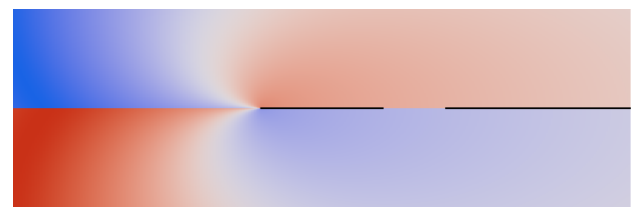

(a) $\max \triangle \mathrm{PWL}$ (noise reduction) - junction 1

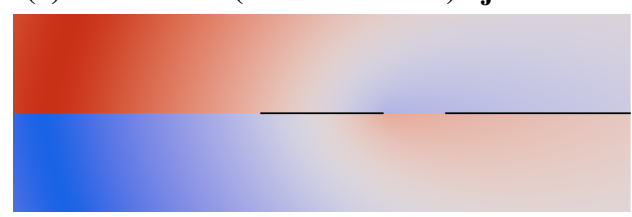

(c) $\max \triangle P W L$ (noise reduction) - junction 2

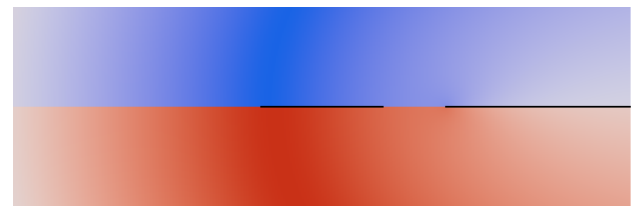

(e) $\max \triangle P W L$ (noise reduction) - junction 3

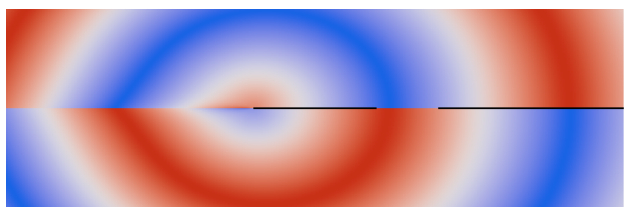

(b) $\min \triangle \mathrm{PWL}$ (noise increase) - junction 1

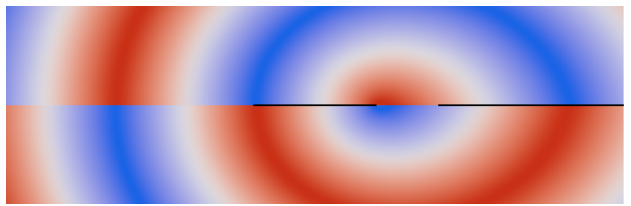

(d) $\min \triangle \mathrm{PWL}$ (noise increase) - junction 2

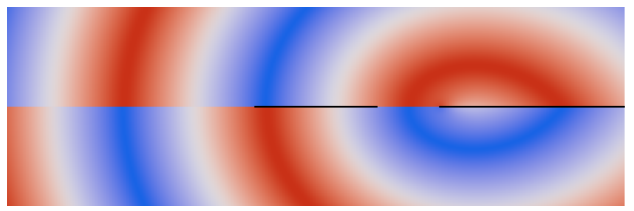

(f) $\min \triangle \mathrm{PWL}$ (noise increase) - junction 3

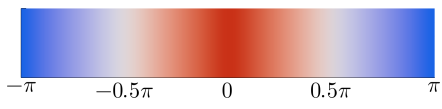

Fig. 6 Instantaneous phase of near field pressure field at frequency corresponding to (a) maximum and (b) minimum $\triangle \mathbf{P W L}$ for $l_{d} / l_{0}=1.5, M=0.1$ 


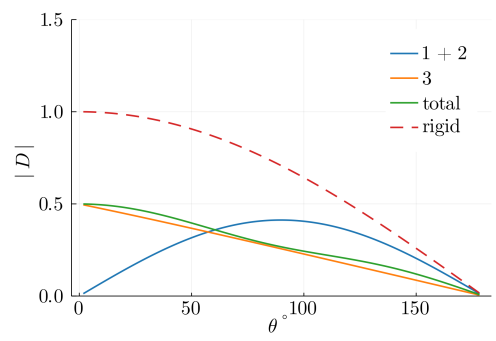

(a) $D_{1}+D_{2}$ compared to $D_{3}$

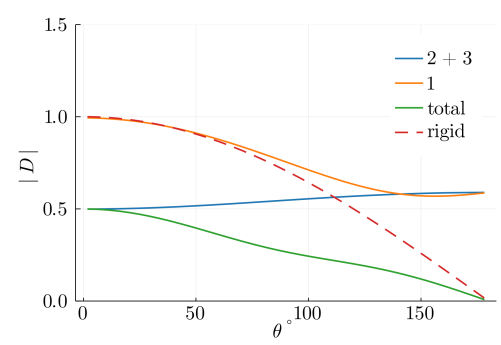

(b) $D_{2}+D_{3}$ compared to $D_{1}$

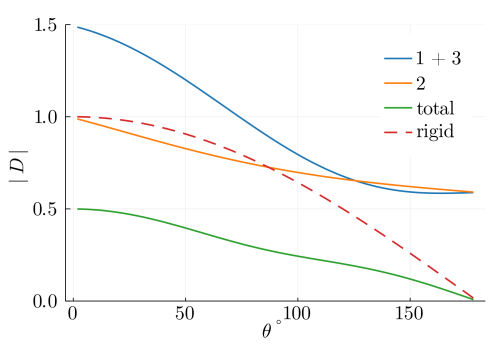

(c) $D_{3}+D_{1}$ compared to $D_{2}$

Fig. 7 Decomposition of the far-field directivity by junction for $M=0.1$ and $l_{d} / l_{0}=1.5$ at maximum in $\triangle \mathrm{PWL}$. Note all values are normalised such that the far-field directivity $D$ for a rigid leading edge at $\theta=0$ is 1 .

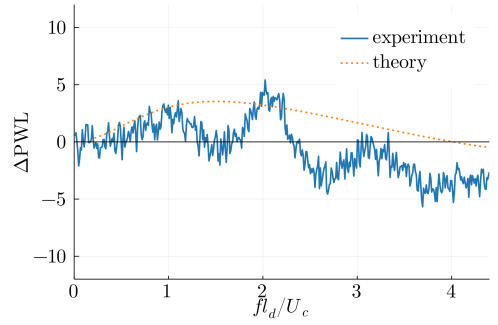

(a) $l_{d} / l_{0}=1.1, U_{\text {jet }}=40 \mathrm{~ms}^{-1}$

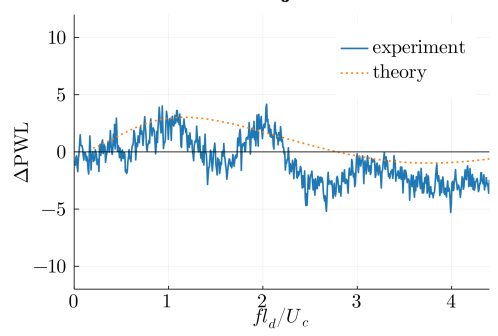

(d) $l_{d} / l_{0}=1.1, U_{\text {jet }}=60 \mathrm{~ms}^{-1}$

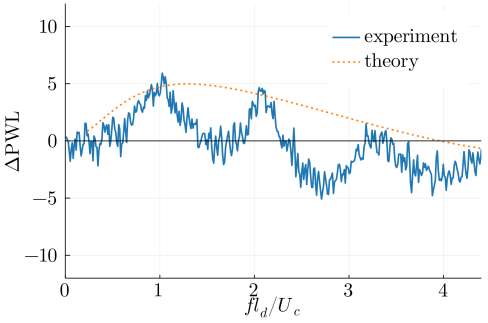

(b) $l_{d} / l_{0}=1.2, U_{\text {jet }}=40 \mathrm{~ms}^{-1}$

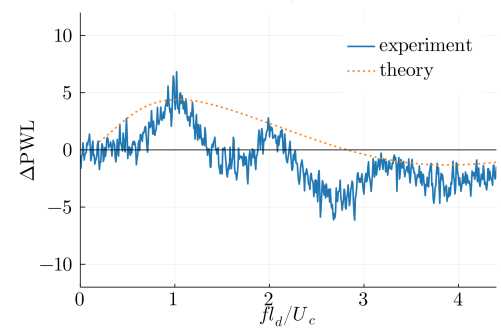

(e) $l_{d} / l_{0}=1.2, U_{\text {jet }}=60 \mathrm{~ms}^{-1}$

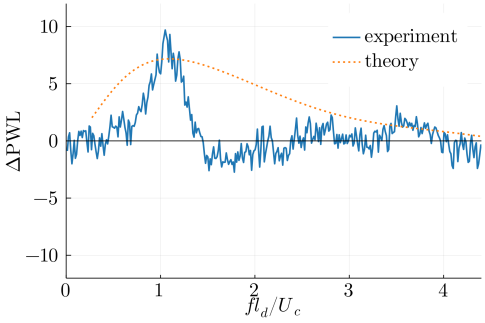

(c) $l_{d} / l_{0}=1.4, U_{\text {jet }}=40 \mathrm{~ms}^{-1}$

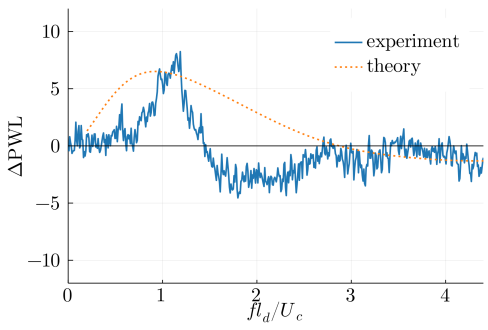

(f) $l_{d} / l_{0}=1.4, U_{\text {jet }}=60 \mathrm{~ms}^{-1}$

Fig. 8 Comparison of $\triangle \mathrm{PWL}$ between theoretical model and wind tunnel observations for experimental cases

angle increases, that at larger observer angles there is a shift to decreasing $\triangle$ SPL and far-field noise reductions occur at lower frequencies. The greater discrepancy at higher frequencies and smaller observer angles is consistent with an increasing importance of other noise sources (e.g. trailing edge noise) and finite chord at these parameters.

Significantly, we do not observe the strong fluctuations in $\triangle \mathrm{PWL}$ that scale on integer values of $f l_{d} / U_{c}$ observed experimentally and are discussed in more detail in [Control ID: 3515636]. The present model is therefore clearly insufficient to describe the experimental findings. It seems plausible that the theoretical model provides an approximate envelope or trend for the noise reductions observed, with a distinct mechanism responsible for these fluctuations. Given the scaling observed, it seems natural to suppose a mechanism associated with junction 3. Within our model framework, this might be investigated by (a) relaxing the unsteady Kutta condition (identifying and scaling the magnitude of the wake), (b) supposing a difference in phase or magnitude of the velocity perturbation incident past junction 3, (c) imposing a leading edge Kutta condition at junction 3 by allowing certain velocity fluctuations, perhaps associated with Tollmien-Schichting waves as has been previously suggested in the literature [18, 19]. Hot-wire measurements should clarify the role of (b), whilst (a) and (c) may be of theoretical interest. A mechanism of the kind (b) underlies the theoretical model postulated in [Control ID: 3515636], where a compact source at junction 3 is supposed to be out of phase with one at junction 1 due to different excitation. It was found that this model is able to account for these fluctuations on $f l_{d} / U_{c}$ at the lowest frequencies of interest. 


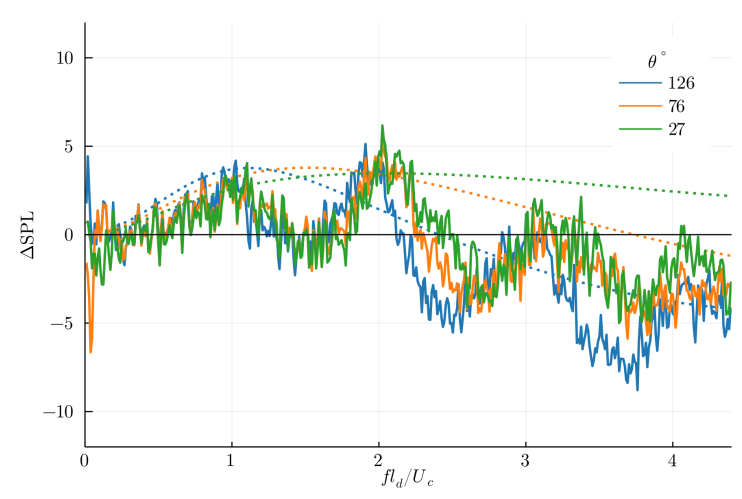

(a) $l_{d} / l_{0}=1.1, U_{\text {jet }}=40 \mathrm{~ms}^{-1}$

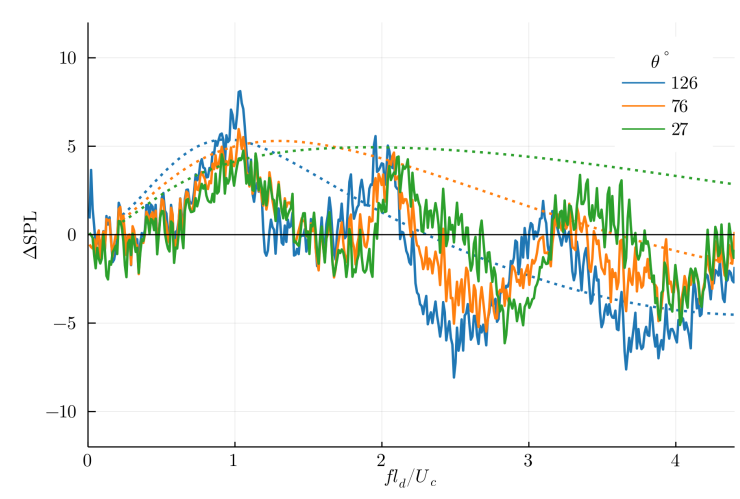

(b) $l_{d} / l_{0}=1.2, U_{\text {jet }}=40 \mathrm{~ms}^{-1}$

Fig. 9 Comparison of far-field SPL variation with observer angle

\section{Conclusion}

In this paper we presented a theoretical study of a leading edge with a slot downstream of the edge, with view to understanding mechanisms for noise reductions associated with introducing perforations near to a leading edge. A Wiener-Hopf problem is formulated for the scattering of an incident gust by a semi-infinite, infinitesimally thin leading edge that is rigid except for a slot of finite length. Across the slot the normal velocity and pressure perturbations are supposed to be continuous. An unsteady Kutta condition is enforced at the upstream edge of the slot, allowing a wake to propagate. The Wiener-Hopf problem was solved by deriving a system of singular integral equations that may be efficiently solved numerically. The model predicts an appreciable reduction in far-field noise at low frequencies, centered approximately on $f l_{d} / U_{c}$, compared to a completely rigid edge. The behaviour is compared with a decomposition of the solution into fields propagating from each junction, and understood as an due to interference between the fields diffracted by the leading edge and the two slot junctions. The model is compared with experimental results from the companion study [Control ID: 3515636], and found to give broad qualitative agreement with their results, providing an approximate envelope for the noise reductions observed. The model in this minimal form is insufficient to replicate oscillations in $\triangle \mathrm{PWL}$ observed experimentally within or from this trend, indicating a distinct or modified mechanism to those included in the model studied here. Future experimental measurements may indicate modifications to the model that could be explored within this framework. The approach employed could offer an extended analysis of the trailing-edge problem studied in [8], and similar multiple scattering problems.

\section{Acknowledgments}

M. J. Priddin acknowledges support from EPSRC DTP EP/N509620/1 and support from Christ's College, University of Cambridge. L. J. Ayton acknowledges support from EPSRC Early Career Fellowship EP/P015980/1. S. Palleja-Cabre would like to acknowledge the financial support of EPSRC (EP/V00686X/1) and P. Chaitanya from the Royal Academy of Engineering (RF/201819/18/194).

\section{A. Numerical solution of the matrix Wiener-Hopf problem}

In this appendix we provide greater detail of the solution of the coupled Wiener-Hopf problem given in section IV

\section{A. Method}

The idea is to work from equation (19) to form three coupled scalar Wiener-Hopf equations. To give a cleaner exposition, we write $\bar{K}=K^{-1}$. We first premultiply the system (19) by $\operatorname{diag}\left(\bar{K}_{-}, \bar{K}_{+}, \bar{K}_{-}\right)$to provide a Wiener-Hopf factorization of the diagonal entries. Here $K=K_{+} K_{-}$and $\bar{K}=\bar{K}_{+} \bar{K}_{-}$denote multiplicative Wiener-Hopf factorizations that may be readily obtained by inspection [11]. This provides a scaled forcing which may be additively factorized

$$
\boldsymbol{G}_{ \pm}=\left(\operatorname{diag}\left(\bar{K}_{-}, \bar{K}_{+}, \bar{K}_{-}\right) \boldsymbol{F}\right)_{ \pm}
$$


We then introduce functions $S_{1}, \ldots, S_{4}$ to give

$$
\begin{array}{ll}
\bar{K}_{-} \Psi_{1-}-G_{1-}+S_{3} & =-K_{+} \Psi_{1+}+G_{1+}-\left(\bar{K}_{-} E_{12+} \Psi_{2+}-S_{3}\right)-K_{+} E_{13+} \Psi_{3+} \\
K_{-} \Psi_{2-}-G_{2-}+\left(\bar{K}_{+} E_{21-} \Psi_{1-}-S_{1}\right)+S_{4} & =-\bar{K}_{+} \Psi_{2+}+G_{2+}-\left(K_{-} E_{23+} \Psi_{3+}-S_{4}\right)-S_{1} \\
\bar{K}_{-} \Psi_{3-}-G_{3-}+\left(K_{+} E_{32-} \Psi_{2-}-S_{2}\right)-\bar{K}_{-} E_{31-} \Psi_{1-} & =-K_{+} \Psi_{3+}+G_{3+}-S_{2}
\end{array}
$$

where $S$ are defined such that bracketed terms on the LHS(RHS) are analytic in, or have analytic continuations with decay at infinity into, the $\mathrm{LH} \alpha \mathrm{P}(\mathrm{UH} \alpha \mathrm{P})$.

The functions $\boldsymbol{S}$ may be explicitly defined as Cauchy transforms along a contour (or union of disjoint contours) that encloses a domain $\mathcal{S}$ containing singularities to be removed, to be evaluated in $\mathbb{C} \backslash \mathcal{S}$. Let $\mathcal{S}(f)$ denote an orientated contour enclosing the singularities of the function $f$, and $\mathcal{C}_{\Gamma}[f](\alpha)$ the Cauchy transform of $f$ along a contour $\Gamma$. We then have

$$
\begin{aligned}
S_{1}(\alpha) & =C_{\mathcal{S}\left(\bar{K}_{+}\right)}\left[\bar{K}_{+} E_{21} \Psi_{1-}\right](\alpha) \\
S_{2}(\alpha) & =C_{\mathcal{S}\left(K_{+}\right)}\left[K_{+} E_{32} \Psi_{2-}\right](\alpha) \\
S_{3}(\alpha) & =C_{\mathcal{S}\left(\bar{K}_{-}\right)}\left[\bar{K}_{-} E_{12} \Psi_{2+}\right](\alpha) \\
S_{4}(\alpha) & =C_{\mathcal{S}\left(K_{-}\right)}\left[K_{-} E_{23} \Psi_{3+}\right](\alpha)
\end{aligned}
$$

Each equation in 277 is arranged such that the LHS(RHS) is analytic in the $\mathrm{LH} \alpha \mathrm{P}(\mathrm{UH} \alpha \mathrm{P})$. By analytic continuation each must define an entire function. We now show that physical conditions on the edge behaviour require that each must vanish identically by analysing the growth of each term. Note that for $M>0$ we have $K_{ \pm} \sim|\alpha|^{ \pm 1 / 2}$, and the Abel theorem states that $\phi(x) \sim x^{\lambda}$ gives $\int_{0}^{\infty} \phi(x) e^{i \alpha x} \mathrm{~d} x \sim|\alpha|^{-1-\lambda}[11,20]$. The growth of $\Psi_{m \pm}$ is so restricted by the asymptotics of the physical field near the junction associated with the algebraic behaviour of $\Psi_{m \pm}$ in $\mathcal{D}_{ \pm}$, i.e. junction $m$. First, we find that each entire function must have at worst algebraic growth, and so by Liouville's theorem, these entire functions must be polynomials. For the leading edge problems, equations $27 \mathrm{a}$ ) and $\left[27 \mathrm{c}\right.$, an entire function $L \sim|\alpha|^{\mu}$ with $\mu=0,1, \ldots$ would be associated with a pressure singularity downstream of the edge of strength $\left|x-x_{\text {edge }}\right|^{-\mu-3 / 2}$. However this singularity must be integrable on physical grounds so $L=0$. For the trailing edge problem, equation [27b), an entire function $L \sim|\alpha|^{\mu}$ would be associated with a pressure singularity $\left|x-x_{\text {edge }}\right|^{-\mu-1 / 2}$ upstream of the edge. The unsteady Kutta condition requires finite pressure, so $L=0$.

We may thus recover expressions for $\boldsymbol{\Psi}_{ \pm}$in terms of the as yet undermined functions $\boldsymbol{S}$. We immediately find that

$$
\begin{aligned}
& \Psi_{1-}=K_{-}\left(G_{1-}-S_{3}\right) \\
& \Psi_{3+}=\bar{K}_{+}\left(G_{3+}-S_{2}\right) \\
& \Psi_{2-}=\bar{K}_{-}\left(G_{2-}-\left(\bar{K}_{+} E_{21-} \Psi_{1-}-S_{1}\right)-S_{4}\right) \\
& \Psi_{2+}=K_{+}\left(G_{2+}-\left(K_{-} E_{23+} \Psi_{3+}-S_{4}\right)-S_{1}\right) \\
& \Psi_{3-}=K_{-}\left(G_{3-}-\left(\bar{K}_{-} E_{12+} \Psi_{2+}-S_{3}\right)-\bar{K}_{-} E_{31-} \Psi_{1-}\right) \\
& \Psi_{1+}=\bar{K}_{+}\left(G_{1+}-\left(K_{+} E_{32-} \Psi_{2-}-S_{2}\right)-K_{+} E_{13+} \Psi_{3+}\right)
\end{aligned}
$$

and after some algebraic simplifications, we have

$$
\begin{aligned}
& \Psi_{1-}=K_{-}\left(G_{1-}-S_{3}\right) \\
& \Psi_{3+}=\bar{K}_{+}\left(G_{3+}-S_{2}\right) \\
& \Psi_{2-}=\bar{K}_{-}\left(G_{2-}-S_{1}+S_{4}\right)+\bar{K}_{+} E_{21-}\left(G_{1-}+S_{3}\right) \\
& \Psi_{2+}=K_{+}\left(G_{2+}-S_{1}+S_{4}\right)+K_{-} E_{23+}\left(G_{3+}+S_{2}\right) \\
& \Psi_{3-}=K_{-}\left(G_{3-}-S_{2}\right) \quad+K_{+} E_{32-}\left(G_{2-}+S_{4}-S_{1}\right) \\
& \Psi_{1+}=\bar{K}_{+}\left(G_{1+}-S_{3}\right) \quad+\bar{K}_{-} E_{12+}\left(G_{2+}-S_{4}+S_{1}\right)
\end{aligned}
$$

These provide expressions for $\boldsymbol{\Psi}$ valid at all points within the intersection of the domains of $\boldsymbol{S}$. We recall that each $S_{m}$ is defined in terms of $\boldsymbol{\Psi}$ evaluated along the boundary of the domain of $S_{m}$. Note that we may choose domains for $\boldsymbol{S}$ such that each domain boundary is contained within the closure of all other domains (this is due to the strip of analyticity). We may combine equations (28) and (29) to formulate a coupled system for the values of $\boldsymbol{\Psi}$ along this boundary, to be 
solved numerically. This may take the form of a singular integral equation

$$
\left(\begin{array}{cccc}
\mathcal{I} & 0 & \mathcal{A}_{13} & 0 \\
\mathcal{A}_{21} & \mathcal{I} & 0 & \mathcal{A}_{24} \\
\mathcal{A}_{31} & 0 & \mathcal{I} & \mathcal{A}_{34} \\
0 & \mathcal{A}_{42} & 0 & \mathcal{I}
\end{array}\right)\left(\begin{array}{l}
\Psi_{1-} \\
\Psi_{2-} \\
\Psi_{2+} \\
\Psi_{3+}
\end{array}\right)=\left(\begin{array}{l}
K_{-} G_{1-} \\
\bar{K}_{-} G_{2-} \\
K_{+} G_{2+} \\
\bar{K}_{+} G_{3+}
\end{array}\right)
$$

where $\mathcal{I}$ is the identity, and the operators $\mathcal{A}_{m n}$ are given by

$$
\begin{aligned}
& \mathcal{A}_{13}[\cdot]=K_{-} C_{\mathcal{S}\left(\bar{K}_{-}\right)}\left[\bar{K}_{-} E_{12+} \cdot\right] \\
& \mathcal{A}_{24}[\cdot]=\bar{K}_{-} C_{\mathcal{S}\left(K_{-}\right)}\left[K_{-} E_{23+} \cdot\right] \\
& \mathcal{A}_{31}[\cdot]=\bar{K}_{+} C_{\mathcal{S}\left(\bar{K}_{+}\right)}\left[\bar{K}_{+} E_{21-} \cdot\right] \\
& \mathcal{A}_{42}[\cdot]=\bar{K}_{+} C_{\mathcal{S}\left(K_{+}\right)}\left[K_{+} E_{32-} \cdot\right]
\end{aligned}
$$

and

$$
\begin{aligned}
& \mathcal{A}_{21}[f](\alpha)=E_{21-}(\alpha) f(\alpha)-\mathcal{A}_{31}[f](\alpha) \\
& \mathcal{A}_{34}[f](\alpha)=E_{23+}(\alpha) f(\alpha)-\mathcal{A}_{24}[f](\alpha)
\end{aligned}
$$

Recall $C$ is the Cauchy operator, $\mathcal{S}(f)$ is the boundary of a domain enclosing the singularities of $f$, we have defined $\bar{K}=K^{-1}$, and $E_{m n \pm}$ are the entries of the matrices $\boldsymbol{E}_{ \pm}$defined in equation 20 . This coupled system may now be solved by introducing bases for a subset of the unknowns $\boldsymbol{\Psi}$ along certain domains required for the evaluation of $\boldsymbol{S}$. Some care is required: note that to evaluate $\mathcal{A}_{21}[f](x)$ we require $f(x)$ and $f\left(\mathcal{S}\left(\bar{K}_{+}\right)\right)$. Since we need the values of $\Psi_{2-}$ at $\mathcal{S}\left(K_{+}\right)$due to the operator $\mathcal{A}_{42}$, we must solve for values of $\Psi_{1-}$ at $\mathcal{S}\left(K_{+}\right)$in addition to values at $\mathcal{S}\left(\bar{K}_{+}\right)$ associated with $\mathcal{A}_{21}$ and $\mathcal{A}_{41}$. Similar considerations should be made for $\mathcal{A}_{34}$, but for the leading edge problem we have $\mathcal{S}\left(K_{-}\right)=\mathcal{S}\left(\bar{K}_{-}\right)$; the domains of the singularities are identical. We remark that we may use the first and last rows of the matrix to eliminate $\Psi_{1-}$ and $\Psi_{3+}$ from rows 2 and 3. One might so first solve for $\Psi_{2 \pm}$, and then recover $\Psi_{1-}$ and $\Psi_{3+}$.

The singularities of $K_{ \pm}, \bar{K}_{ \pm}$are branch cuts along rays with square root endpoint singularities, and $K_{+}$possesses a single pole. We deform the contours $\mathcal{S}(f)$ onto the enclosed singularities: for poles we choose a small circle enclosing only the pole, and for branch cuts we deform precisely onto the branch cut, giving a single Cauchy integral of the jump across the cut. The jump along the branch cuts decay exponentially (recall we have chosen branch cuts to be parallel to the imaginary axis), so we may truncate the contour at a finite value where the jump is numerically zero.

We now pose basis expansions for $\Psi_{1-}, \Psi_{2-}, \Psi_{2+}, \Psi_{3+}$ along the contours where their values are required. Since each is analytic on the contours, standard approximation schemes may be applied and are anticipated to provide spectral accuracy. For circular domains, we take a Taylor expansion, for which the argument of the Cauchy transform may be represented by a Laurent expansion. It can be permissible to reduce the radius of the domain to zero and only retain the first coefficient, in which case the method reduces to a standard application of pole removal. Chebyshev polynomials are used to represent the values of $\boldsymbol{\Psi}$ along line segments; the argument of the Cauchy transforms may then be represented as Chebyshev polynomials against the square root weight $\left(1-x^{2}\right)^{-1 / 2}$. The square root behaviour at -1 enables us to precisely capture jump across the branch cuts at $\pm \hat{k}$. The exponential decay of the jump away from $\pm \hat{k}$ leads to rapid decay of function values along the cut; this appears to make the precise behaviour of the weight function at $x=1$, corresponding to the point at which the branch cut domain is truncated, unimportant.

By choosing the above representation spaces for the domain, and choosing the range space for segments to be Chebyshev polynomials of the second kind, the total linear operator A in coefficient space is banded in finite precision, that is

$$
\forall \varepsilon>0 \quad \exists m<\infty \text { s.t. } \forall|i-j|>m \quad\left|\mathrm{~A}_{i j}\right|<\varepsilon
$$

This follows from the following points. First, the Cauchy operator between a domain and range separated by a finite distance being compact. Second, the Hilbert transform (for evaluating the limiting values of the Cauchy integral on the domain) acting on a Laurent space, or from Chebyshev first kind polynomials against $\left(1-x^{2}\right)^{-1 / 2}$ to Chebyshev second kind polynomials, are banded. Lastly, multiplication on these spaces are banded operators in coefficient space, as is conversion from Chebyshev first kind polynomials to Chebyshev second kind polynomials.

This approach of generating banded operators in coefficient space was developed in [16] and implemented in the Julia package ApproxFun.j1 [21]. The development of the approach to singular integral equations was given in [17] and 
implemented in the Julia package SingularIntegralEquations.jl. Work to fully embed this method into this numerical framework for infinite dimensional linear algebra using ApproxFun.jl is ongoing, so far implemented for $M=0$ when no poles exist in $K$. For simplicity we solve a truncated system in the least squares sense, checking convergence with truncation.

We may perform numerical checks on the approach by ensuring continuity across removed branch cuts (up to a prescribed tolerance) and computing the residue about removed poles appearing in the expressions (30). We may also verify the later numerical inversion provides a continuous function satisfying the correct boundary conditions and edge behaviour at $y=0$ (the radiation condition is satisfied immediately).

\section{References}

[1] Geyer, T., “Trailing edge noise generation of porous airfoils,” doctoralthesis, BTU Cottbus - Senftenberg, 2011.

[2] Roger, M., Schram, C., and Santana, L. D., "Reduction of Airfoil Turbulence-Impingement Noise by Means of Leading-Edge Serrations and/or Porous Material," 19th AIAA/CEAS Aeroacoustics Conference, 2013. doi:10.2514/6.2013-2108.

[3] Priddin, M. J., Paruchuri, C. C., Joseph, P., and Ayton, L. J., "A Semi-analytic and Experimental Study of Porous Leading Edges," 25th AIAA/CEAS Aeroacoustics Conference, 2019, p. 2552.

[4] Jaworski, J., and Peake, N., "Aerodynamic noise from a poroelastic edge with implications for the silent flight of owls," Journal of Fluid Mechanics, Vol. 723, 2013, p. 456-479. doi:10.1017/jfm.2013.139.

[5] Lyu, B., and Azarpeyvand, M., "On the noise prediction for serrated leading edges," Journal of Fluid Mechanics, Vol. 826, 2017, p. 205-234. doi:10.1017/jfm.2017.429.

[6] Ayton, L. J., and Kim, J. W., "An analytic solution for the noise generated by gust-aerofoil interaction for plates with serrated leading edges," Journal of Fluid Mechanics, Vol. 853, 2018, p. 515-536. doi:10.1017/jfm.2018.583.

[7] Chaitanya, P., and Joseph, P., "Slitted leading edge profiles for the reduction of turbulence-aerofoil interaction noise," The Journal of the Acoustical Society of America, Vol. 143, No. 6, 2018, pp. 3494-3504. doi:10.1121/1.5040972.

[8] Howe, M. S., and Lighthill, M. J., "Aerodynamic sound generated by a slotted trailing edge," Proceedings of the Royal Society of London. A. Mathematical and Physical Sciences, Vol. 373, No. 1753, 1980, pp. 235-252. doi:10.1098/rspa.1980.0146.

[9] Crighton, D. G., "The Kutta condition in unsteady flow," Annual Review of Fluid Mechanics, Vol. 17, No. 1, 1985 , pp. 411-445.

[10] Ayton, L. J., Gill, J. R., and Peake, N., "The importance of the unsteady Kutta condition when modelling gust-aerofoil interaction," Journal of Sound and Vibration, Vol. 378, 2016, pp. 28-37.

[11] Noble, B., Methods based on the Wiener-Hopf technique for the solution of partial differential equations, Chelsea Pub Co, 1988.

[12] Kisil, A. V., "An Iterative Wiener-Hopf Method for Triangular Matrix Functions with Exponential Factors," SIAM Journal on Applied Mathematics, Vol. 78, No. 1, 2018, pp. 45-62. doi:10.1137/17M1136304.

[13] Priddin, M. J., Kisil, A. V., and Ayton, L. J., "Applying an iterative method numerically to solve $n \times n$ matrix Wiener-Hopf equations with exponential factors," Philosophical Transactions of the Royal Society A: Mathematical, Physical and Engineering Sciences, Vol. 378, No. 2162, 2020, p. 20190241. doi:10.1098/rsta.2019.0241.

[14] Nigro, D., "Prediction of broadband aero and hydrodynamic noise: derivation of analytical models for low frequency," Ph.D. thesis, University of Manchester, 2017.

[15] Hirschberg, A., and Rienstra, S. W., “An Introduction to Aeroacoustics,” Tech. rep., 2004.

[16] Olver, S., and Townsend, A., "A fast and well-conditioned spectral method," SIAM Review, Vol. 55, No. 3, 2013 , pp. 462-489.

[17] Slevinsky, R. M., and Olver, S., "A fast and well-conditioned spectral method for singular integral equations," Journal of Computational Physics, Vol. 332, 2017, pp. 290-315.

[18] Job, S., Lunéville, E., and Mercier, J.-F., "Diffraction of an acoustic wave by a plate in a uniform flow: a numerical approach," Journal of Computational Acoustics, Vol. 13, No. 04, 2005, pp. 689-709.

[19] Howe, M., "The role of displacement thickness fluctuations in hydroacoustics, and the jet-drive mechanism of the flug organ pipe," Proceedings of the Royal Society of London. A. Mathematical and Physical Sciences, Vol. 374, No. 1759, 1981, pp. 543-568. 
[20] Crighton, D., and Leppington, F., "Scattering of aerodynamic noise by a semi-infinite compliant plate," Journal of Fluid Mechanics, Vol. 43, 1970, p. 721-736. doi:10.1017/S0022112070002690.

[21] Olver, S., and Townsend, A., "A practical framework for infinite-dimensional linear algebra," Proceedings of the 1st Workshop for High Performance Technical Computing in Dynamic Languages - HPTCDL '14, IEEE, 2014. 